\title{
P02-12
}

\section{THE ROLE OF QUANTITATIVE ELECTROENCEPHALOGRAPHY IN PSYCHIATRY}

T. Rodrigues, I. Saavedra

Hospital de Magalhães Lemos, Porto, Portugal

Background: The more recent advances in the field of Neurosciences include the study of abnormal pathophysiological findings in patients suffering from Psychiatric disorders. Several new brain imaging technologies are conveying numerous data concerning structural and functional abnormalities in such patients.

Aims: To provide an overview of the role of quantitative Electroencephalography (qEEG) in Psychiatry.

Methods: Review of the literature.

Results: Among the various imaging studies, the application of three-dimensional qEEG may be the most practical and economic alternative. The qEEG consists in the statistical analysis of the EEG parameters, with computer-treated data. It is a portable, radiation-free, non-invasive method that measures excitatory and inhibitory cortical neuronal activity directly.

Conclusion: The latest literature regarding the potential role of the qEEG in Psychiatry debates its applicability in clinical settings. Several authors have been trying to evaluate its usefulness in clinical diagnosis and prediction of response to medication. We review the possible recommendations for the use of this test and the controversies surrounding them. 